\title{
L. Poort, Consensus and Controversies in Animal Biotechnology. An Interactive Legislative Approach to Animal Biotechnology in Denmark, Switzerland, and the Netherlands
}

\section{A. Daniel Oliver-Lalana}

\section{Poort, Consensus and Controversies in Animal Biotechnology. An Interactive Legisla-} tive Approach to Animal Biotechnology in Denmark, Switzerland, and the Netherlands (The Hague: Eleven, 2013), 186 p.

In the last decades a variety of approaches to legislation and regulation has spread which attempt to keep pace with increasingly complex social environments. What unites them is the thesis that cybernetic and rule-based modes of lawgiving - often associated with legal instrumentalism - are no longer, at least not always, adequate to govern society. Interactive and communicative theories are well to the fore in this strand; loosely speaking, they submit that laws are likely to be (more) legitimate and effective when both its formation and application occur through a collaborative and continued effort between all relevant actors and stakeholders - whereby standards, principles or symbolic norms fulfil a crucial function. This strategy is frequently claimed to be best suited for regulating emergent issues with deep moral implications. Hence the use of animals for biotechnological research and experimentation offers an optimal field for testing the core tenets of interactive legislation and exploring its virtues and shortcomings. And that is precisely what Lonneke Poort's study does: it articulates the basic features of the interactive approach into an ideal-typical model, contrasts it with animal biotechnology regulations in Denmark, Switzerland and the Netherlands, and leans on these national examples to reconsider some interactivist principles, especially the orientation towards consensus - a controversial ethos is suggested instead as the key for boosting dynamic processes of legal norm development.

Poort's starting idea is that the rapid, uncertain advance of animal biotechnology, along with the 'intractable' value disagreements over its limits, makes clear-cut legal intervention not only hard to define, but also inappropriate: prospects of regulatory success improve if a flexible, interactive strategy is adopted. Yet since there are different understandings of this strategy, the author first sets out to reconstruct its essentials. Drawing mainly on Van der Burg and Brom's account - Black's facilitative regulation and Brunee and Toopes' theory of international law are considered as well - , she delineates a framework for analysis that stresses the cooperative and dynamic components of interactive legislation. In a nutshell, cooperation implies ample social participation in all stages of the regulatory process, while dynamics is reflected in the preference for open norms stimulating 
ongoing processes of norm development where moral values play a prominent part and consensus serves as a guiding aspiration. Interactive legislation would thus imply the 'mutual reinforcement' of moral and legal discourses. With an eye on the 'quality' requirements for law in general - also tackled in the book -, Poort rounds off her model with three hypotheses: this legislative approach is expected to live up to requirements of both democratic and substantive legitimacy and to cope with problems in the long run without necessarily fixing a definite solution.

Referring to this framework, the author analyzes to what extent animal biotechnology regulations in Denmark, Switzerland and the Netherlands meet the criteria of interactive legislation. After giving an overview of the legislative background, the relevant legal texts and the licensing procedure in each country, she concentrates on the public involvement and the shaping of moral values (e.g., 'animal integrity,' 'dignity of living beings,' 'intrinsic value of animals') throughout the regulatory process, and examines the type of legitimacy underlying the legal regime - popular participation or substantive justification 'in light of principles, ideals and policies.' Special attention is thereby paid to the role of ethics committees. In addition, by delving into the political and the legal culture of the countries upon focus, Poort tries to identify the conditions under which interactive legislation may succeed - for example, this approach would be particularly illmatched with legal formalism and positivistic jurisprudential traditions. Overall, her case studies yield split findings: whereas claims related to interactivity, public participation and popular legitimacy are more or less corroborated in all three countries (with certain reservations about Switzerland), those about dynamics are not, or not sufficiently: even when open norms were used which could have fuelled deliberation in the implementation phase, ongoing processes of norm development were not established, which make the hypotheses on substantive legitimacy and long-run problem-solving fail.

Poort blames these deficiencies on two major factors: the emphasis on consensus and the legalistic application of animal biotechnology law. On the one hand, when it comes to regulate issues pervaded by intractable disagreements, an ethos of consensus would prove counterproductive: licensing decisions are taken as if they were backed by shared understandings that don't actually exist, and such a fictional consensus precludes further reasoning and leads to the 'stagnation' of the process of norm development - the regulatory design may have pursued the opposite, though. On the other, Poort's analysis of the application of the Danish, Swiss, and Dutch laws reveals that the legalistic environment in which decisionmakers operate hampers moral debates - this also holds for legal cultures prone to interactionism: instead of a mutual reinforcement, moral norm development was sacrificed for the sake of settling the law. Accordingly, two central pieces of the interactive model fall short in practice. So Poort suggests an alternative conception which may preserve legislative dynamics by replacing consensus with an 'ethos of controversies' and by dissociating legal and moral norm development in a 'two-track approach.' Since dissent can't be banished from morally delicate issues like animal biotechnology, regulation should achieve the maximum possible level of inclusiveness. Instead of focusing on consensus - which leads to silencing some legitimate voices - value conflicts should, rather, be kept visible all 
along the regulatory cycle, even after concrete legal decisions have been taken. This way, dissenting viewpoints not only get duly recognized, but also can contribute to rethink the problems at stake and could eventually be reintroduced into the legal discourse. In short: for Poort, the controversial ethos fosters norm (re-)construction and assures an inclusive representation of all values before, during and after the decision making process. Yet, for this ethos to function properly, a two-track model is needed which keeps the legal and the moral apart: what is legally settled and what is morally correct must be separated. The interactive legislative approach should therefore be centred only on the development of legal norms; moral norms - Poort concludes - should be developed in a different track that is not bound by legal restrictions.

Although the book presents a sound and thorough case for dissent-respecting interactive law, some objections might be posed. To start with, neither interactive nor clear-cut legislation are feasible strategies when conceived in pure form: the heart of the matter is, rather, how they combine - a question that is barely thematized. The presence of certain 'legalistic' or 'instrumentalist' features within a largely interactive regulation process doesn't render it flawed. Probably, the practicability and empirical adequacy of an ideal-typical model is already evidenced when 'most of [their] tenets are recognizable' (p. 117), as it happens in the Dutch case. A second, related aspect is that Poort refrains from taking stance on the quality of the regulations examined. Beyond implicit criticisms, she is primarily concerned with testing her own interactive legislation scheme, i.e., with discussing to what extent those regulations conform to it. She doesn't evaluate whether the legislative strategies adopted in the case study countries proved reasonable or had positive impacts. Whether they deserve an 'interactive' label or not is, in the end, ancillary to their being right strategies. Thirdly, Poort's model remains 'domain-specific,' both in its original (p. 39, 44) and amended version: the controversial ethos applies just where intractable disagreements exist and the two-track proposal is restricted to issues like animal biotechnology (p. 165, $159 \mathrm{n} 23$ ), which leaves open the question whether interactive legislation will work out in other contexts. Finally, it would have been desirable to have more samples of arguments (e.g., about animal dignity and integrity) justifying the granting or denial of licenses for animal testing; for it is not apparent how the legal colonization of moral norm development occurred - neither is Poort's 'separation thesis' very easy to grasp. For instance, I fail to see why resorting to the principle of proportionality diminishes the scope for moral deliberation or can't be reconciled with an interactive approach.

None of this, however, outweighs the merits of the book, which not only makes a contribution to the theory of interactive legislation, but also includes a key component of empirical, applied research and a valuable comparative perspective on the regulatory regimes and practices in the domain of animal biotechnology. Furthermore, the author aptly completes this analysis by appraising how the specific traits of the political-democratic and legal culture constitute the framework within which interactive legislation may (not) thrive. All in all, Poort's study provides an insightful illustration of the interactive approach, shows where its main pitfalls lay and how they could be surmounted. This makes it especially interest- 
ing to legislation or regulation theorists, as well as to bioethicists and scholars occupied with animal biotechnology law; and also general readers from the fields of legal and political sciences might benefit from the book, for it offers a practical introduction to the thorny task of legislation and regulation in society today. This cannot be but welcome. 